\title{
Design of controllers for higher order systems
}

\author{
Andrey Prokopev \\ School of Engineering and \\ Construction \\ Siberian Federal University \\ Krasnoyarsk, Russia \\ prok1@yandex.ru
}

\author{
Zhasurbek Nabizhanov \\ School of Space and Information \\ Technology \\ Siberian Federal University \\ Krasnoyarsk, Russia \\ jasur150691@yandex.ru \\ Rurik Emelyanov \\ School of Engineering and \\ Construction \\ Siberian Federal University \\ Krasnoyarsk, Russia \\ ert-44@yandex.ru
}

\author{
Vladimir Ivanchura \\ School of Space and Information \\ Technology \\ Siberian Federal University \\ Krasnoyarsk, Russia \\ ivan43ura@yandex.ru
}

\begin{abstract}
A method of parametric synthesis of the PID controller of the control system for high-order objects based on the modal method for linear systems with the location of complex-conjugate poles is proposed. The methodology provides for inspection of the controller parameters according to the algebraic criterion of stability of Routh-Hurwitz to change all positive values. On the example of designing a PID controller of the control system for the object of the fourth order, the efficiency of the technique is demonstrated
\end{abstract}

Keywords-parametric synthesis, automatic control system, PID controller, modal method, poles, transient characteristic

\section{INTRODUCTION}

The spread of information modeling technologies for construction project (conception of BIM-technologies) contributes to the development of automatic control system in the road construction $[1,2]$.

From scientific sources it is possible to single out methods of the synthesis of PID regulators [2-11]: empiric tuning; Ziegler-Nichols methods and derived methods; algebraic method; methods of modal synthesis; methods of frequency domain synthesis; method of optimal synthesis; method of optimal trunsfer function in closed system (technical and symmetric optima). The main trends in the development of methods for the synthesis of PI and PID regulators are discussed in Aidan O'Dwyer, 2006 [9] (443 methods for the synthesis of PID regulators) and in Aidan O'Dwyer, 2009 [10] (1731 methods for the synthesis of PID regulators).

Among the methods of synthesis of PID-regulators developed by scientists, is the modal method $[5,11]$. Synthesis of controllers for high order objects (above second) is actual. Mathematical models of working processes of road and construction machines as control object are described by differential equation and transfer function of second or higher order [12-19].

\section{INITIAL MATHEMATICAL FORMULATION OF SYSTEM AND RESEARCH METHODOLOGIES}

We consider a linear automatic control system with trunsfer function (TF) of an object higher than the second order.

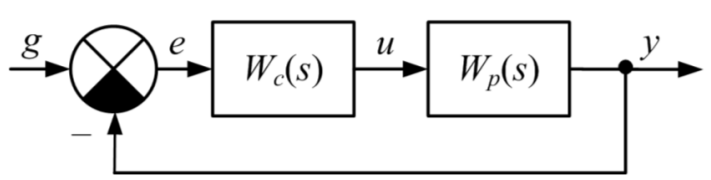

Fig. 1. Control system structure

Transfer function of high-order control objects $W_{p}(s)$ and PID-controller $W_{c}(s)$ have the following form:

$$
\begin{gathered}
W_{p}(s)=\frac{b_{m} \cdot s^{m}+b_{m-1} \cdot s^{m-1}+\ldots+b_{0}}{a_{n} \cdot s^{n}+a_{n-1} \cdot s^{n-1}+\ldots+a_{0}} \\
=\frac{N(s)}{D(s)}, \text { where } m<n, \\
W_{c}(s)=K_{P}+\frac{K_{I}}{s}+K_{D} \cdot s \\
=\frac{K_{D} \cdot s^{2}+K_{P} \cdot s+K_{I}}{s} .
\end{gathered}
$$

Where $s$ - Laplace operator; $K_{P}, K_{I}, K_{D}$ coefficients of, respectively, proportionality, integration and differentiation.

Due to series coupling of PID-controller and control object they form open loop system with transfer function of the following type

$$
W(s)=W_{c}(s) \cdot W_{p}(s) .
$$

Transfer function of closed control system with negative unity feedback

$$
K(s)=\frac{W(s)}{1+W(s)}
$$

The initial data for the parameters analytical synthesis of the PID controller of automatic control system are a model of the transfer function controller, the transfer function of the 
high-order control object $W_{p}(s)$ and the specified quality indicators: excessive correction and response time.

The synthesis problem of parameters $K_{P}, K_{I}, K_{D}$ PID controller of closed control that provides specified quality indicators when operating a control object with the high order TF (higher than the second one) $W_{p}(s)$ by the modal method for the variant of specifying the number and type of roots of the characteristic polynomial of this system is solved: the dominant real pole $s_{1}=-\eta_{1}$ and complex poles

$$
\begin{aligned}
& s_{2}=-\eta_{2}+j \cdot \beta_{2}, s_{3}=-\eta_{2}-j \cdot \beta_{2}, \ldots, \ldots, \\
& s_{n}=-\eta_{n}+j \cdot \beta_{n}, s_{n}=-\eta_{n}-j \cdot \beta_{n} .
\end{aligned}
$$

The algorithm of the methodology includes the following (see below) steps.

Step 1: By the known transfer function (TF) $W_{p}(s)$ of the control object (1) and the PID controller $W_{c}(s)$ (2) with unknown coefficients $K_{P}, K_{I}, K_{D}$, the $\mathrm{TF}$ of the open system is determined:

$$
\begin{aligned}
W(s) & =W_{c}(s) \cdot W_{p}(s) \\
= & \frac{B\left(K_{P}, K_{I}, K_{D}, s\right)}{C(s)} .
\end{aligned}
$$

Step 2: The structure of the polynomial and the polynomial of the closed system are determined by adding the numerator $B\left(K_{P}, K_{I}, K_{D}, S\right)$ with unknown coefficients $K_{P}, K_{I}, K_{D}$ and the denominator $C(s)$

$$
\begin{aligned}
& D\left(K_{P}, K_{I}, K_{D}, s\right) \\
& \quad=B\left(K_{P}, K_{I}, K_{D}, s\right)+C(s) .
\end{aligned}
$$

Step 3: The values of the complex poles

$$
\begin{aligned}
& s_{2}=-\eta_{2}+j \cdot \beta_{2}, s_{3}=-\eta_{2}-j \cdot \beta_{2}, \ldots, \ldots, \\
& s_{n}=-\eta_{n}+j \cdot \beta_{n}, s_{n}=-\eta_{n}-j \cdot \beta_{n} .
\end{aligned}
$$

The values of the poles for providing basic quality indicators of the transient process are assigned in the region bounded by the lines in Fig. 2. This condition is connected with the fact that indirect estimates of the quality of the transient process are used - root indicators, by the form and location of the roots $s_{1}, s_{2}, \ldots, s_{n}$ of the characteristic equation of a closed loop $a_{n} \cdot s^{n}+a_{n-1} \cdot s^{n-1}+\ldots+a_{0}=0$ on the complex plane.

In Fig. 2 the following symbols are used: $\eta$ - degree of stability, $\varphi$ - angle, its maximum value is used to determine the root quality of the degree of oscillatory system $\mu=\operatorname{tg} \varphi_{\max }, \xi-$ distance from the imaginary axis to the most remote root (roots).

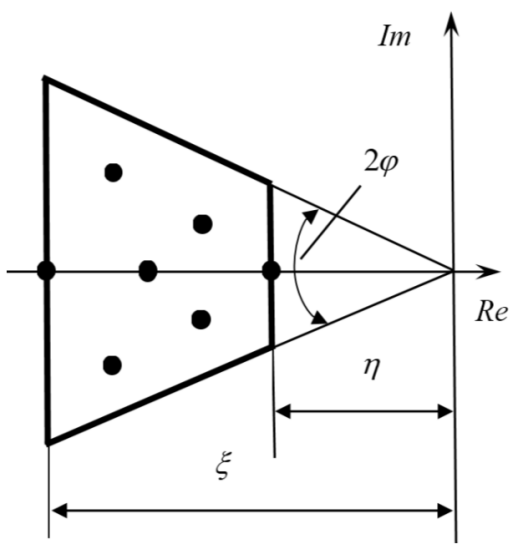

Fig. 2. Root quality data

The main influence on the nature of the transient process of the control system is exerted by the dominant roots located closer to the imaginary axis, which give the longest components of the transient process [8, 20-22]. If the nearest root is real (see Fig. 2, root $s_{1}$ ), then the dominant component of the transient process will be an exponent with exponent $s_{k}=-\eta$ :

$$
x_{k}(t)=C_{k} \cdot e^{-\eta \cdot t}
$$

If two conjugate complex roots are closest to the imaginary axis, then one vibrational component will dominate, which also decays according to the exponential component (4).

In both cases, the duration of the transient process $t_{p}$ is determined by the approximate formula [7, 17, 18, and 19]:

$$
t_{p} \leq 3 / \eta
$$

where the equality sign refers to the case of the actual dominant root, and the inequality sign to the case of complex dominant roots.

Step 4: The characteristic polynomial of a closed loop is defined in the target function:

$$
\begin{aligned}
& D\left(s_{1}, s_{2}, \ldots, s_{n}, s\right) \\
& \quad=\left(s-s_{1}\right)\left(s-s_{2}\right) \ldots\left(s-s_{n}\right) .
\end{aligned}
$$

Step 5: To Parameters $K_{P}, K_{I}, K_{D}$ from algebraic equations are calculated equating expressions (3) and (5). Check one of the two conditions for implementing the algorithm of the technique in question - the values of the PID regulator coefficients should be positive, otherwise, point 3 of the technique with the choice of the other roots $s_{1}, s_{2}, s_{3}, s_{4}, s_{5}$ of the characteristic equation of the closed system is repeated. 
Step 6: By the characteristic polynomial (3) of the closed control system, the stability of the automatic control system is tested using the algebraic Routh stability criterion. If stability is not ensured, point 3 of the procedure with a choice of other values of the roots $s_{1}, s_{2}, \ldots, s_{n}$ of the characteristic equation of the system is repeated.

\section{INTERPRETATION AND DISCUSSION OF RESEARCH RESULTS}

Example for complex pole case. Initial data for the design task for the PID controller of automatic control system: response time $t_{p}=0.02 \mathrm{~s}$; transient without overshooting.

The transfer function of the control object of the 4th order $W_{p}(s)$ is defined, consisting of two consecutive blocks $W_{1}(s)$ and $W_{2}(s)$, Fig. 3

$$
\begin{aligned}
& W_{1}(s)=\frac{1}{\left(T_{G P} \cdot s+1\right) \cdot\left(T_{M}{ }^{2} \cdot s^{2}+2 \cdot \xi_{M} \cdot T_{M} \cdot s+1\right)} \\
& W_{2}(s)=\frac{1}{T \cdot s+1} ; W_{p}(s)=W_{1}(s) \cdot W_{2}(s) .
\end{aligned}
$$

The following values of the variables included in the TF are used (comparable to the real ones):

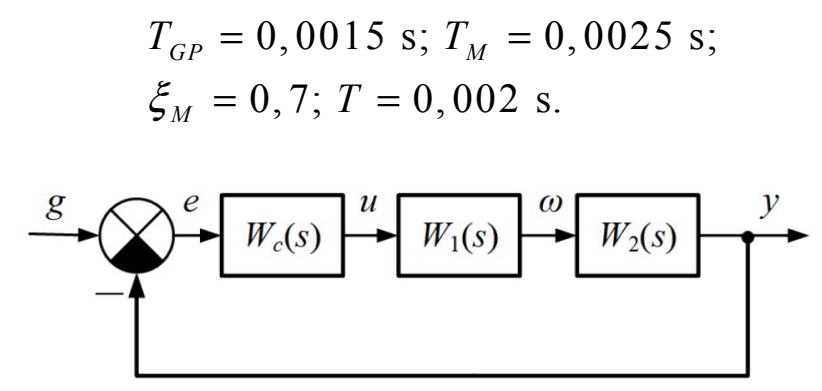

Fig. 3. Schematic structure of synthesizable automatic control system

The structure of the transfer function of the PID controller $W_{c}(s)$ is set in accordance with the formula (2). It is required to determine the parameters of the PID controller $K_{P}, K_{I}, K_{D}$ on the basis of algorithm of the analytical synthesis considered in this paper.

Let us determine the total TF $W(s)$ of the open system (Fig. 1, Fig. 2):

$$
\begin{gathered}
W(s)=W_{c}(s) \cdot W_{p}(s)=\frac{B\left(K_{P}, K_{I}, K_{D}, s\right)}{C(s)} \\
=\frac{1,6 \cdot 10^{11}\left(K_{D} \cdot s^{2}+K_{I} \cdot s+K_{P}\right)}{\left[\begin{array}{c}
\left.3 \cdot s^{5}+5180 \cdot s^{4}+3,44 \cdot 10^{6} \cdot s^{3}\right] \\
\left.+1,12 \cdot 10^{9} \cdot s^{2}+1,6 \cdot 10^{11} \cdot s\right]
\end{array}\right.}
\end{gathered}
$$

We obtain a polynomial of the closed loop by adding the denominator $C(s)$ and the numerator $B\left(K_{P}, K_{I}, K_{D}, s\right)$ of the TF of the open system:

$$
\begin{aligned}
D( & \left.K_{P}, K_{I}, K_{D}, s\right)=C(s) \\
& +B\left(K_{P}, K_{I}, K_{D}, s\right)=3 \cdot s^{5}+5180 \cdot s^{4} \\
& +3,44 \cdot 10^{6} \cdot s^{3} \\
& +\left(1,12 \cdot 10^{9}+1,6 \cdot 10^{11} \cdot K_{D}\right) \cdot s^{2} \\
& +\left(1,6 \cdot 10^{11}+1,6 \cdot 10^{11} \cdot K_{I}\right) \cdot s \\
& +1,6 \cdot 10^{11} \cdot K_{P} .
\end{aligned}
$$

We perform the transformation of the polynomial (6) by reducing the element with the highest degree to 1 :

$$
\begin{aligned}
D( & \left.K_{P}, K_{I}, K_{D}, s\right)=s^{5}+1726,7 \cdot s^{4} \\
& +1,147 \cdot 10^{6} \cdot s^{3}+\left(5,3 \cdot 10^{10} \cdot K_{D}\right. \\
& \left.+3,73 \cdot 10^{8}\right) \cdot s^{2}+\left(5,3 \cdot 10^{10} \cdot K_{P}\right. \\
& \left.+5,3 \cdot 10^{10}\right) \cdot s+5,3 \cdot 10^{10} \cdot K_{I}
\end{aligned}
$$

For convenience, we represent equation (7) in the form of a column vector $b_{1}$ :

$$
b_{1}=\left[\begin{array}{l}
5,3 \cdot 10^{10} \cdot K_{I} \\
5,3 \cdot 10^{10} \cdot K_{P}+5,3 \cdot 10^{10} \\
5,3 \cdot 10^{10} \cdot K_{D}+3,73 \cdot 10^{8} \\
1,147 \cdot 10^{6} \\
1726,7 \\
1
\end{array}\right] .
$$

We give the values of the roots $s_{1}, s_{2}, s_{3}, s_{4}, s_{5}$ of the characteristic equation of a closed loop with allowance for placement in the recommended region, see Fig. 2, based on the practice of designing automatic control systems.

An arbitrary function with an identical degree is defined and the elements are grouped in increasing degree:

$$
\begin{aligned}
D( & \left.s_{1}, s_{2}, s_{3}, s_{4}, s_{5}, s\right)=\left(s-s_{1}\right) \cdot\left(s-s_{2}\right) \\
& \cdot\left(s-s_{3}\right) \cdot\left(s-s_{4}\right) \cdot\left(s-s_{5}\right) \\
& =s^{5}+\left[-s_{1}-s_{2}-s_{3}-s_{4}-s_{5}\right] \cdot s^{4} \\
& +\left[\mathrm{S}_{4} \cdot\left(\mathrm{S}_{1}+\mathrm{S}_{2}+\mathrm{S}_{3}\right)+s_{5} \cdot\left(s_{1}+s_{2}\right.\right. \\
& \left.\left.+s_{3}+s_{4}\right)+s_{3} \cdot\left(s_{1}+s_{2}\right)+s_{1} \cdot s_{2}\right] \cdot s^{3} \\
& +\left[-s_{5} \cdot\left(s_{4} \cdot\left[s_{1}+s_{2}+s_{3}\right]+s_{3} \cdot\left[s_{1}+s_{2}\right]\right.\right. \\
& \left.+s_{1} \cdot s_{2}\right)-s_{4} \cdot\left(s_{3} \cdot\left[s_{1}+s_{2}\right]+s_{1} \cdot s_{2}\right) \\
& \left.-s_{1} \cdot s_{2} \cdot s_{3}\right] \cdot s^{2}+\left[s _ { 5 } \cdot \left(s _ { 4 } \cdot \left[s_{3} \cdot\left(s_{1}+s_{2}\right)\right.\right.\right. \\
& \left.\left.\left.+s_{1} \cdot s_{2}\right]+s_{1} \cdot s_{2} \cdot s_{3}\right)+s_{1} \cdot s_{2} \cdot s_{3} \cdot s_{4}\right] \\
& \cdot s-s_{1} \cdot s_{2} \cdot s_{3} \cdot s_{4} \cdot s_{5} .
\end{aligned}
$$


In this example, the following roots are taken

$$
\begin{aligned}
& s_{1}=-325+j \cdot 163 ; s_{2}=-325-j \cdot 163 \\
& s_{3}=-335+j \cdot 265 ; s_{4}=-335-j \cdot 265 \\
& s_{5}=-355
\end{aligned}
$$

After substituting the values of the roots of the emotional control of a closed loop in the system (8), we obtain a column vector $b_{2}$ with specific values

$$
b_{2}=\left[\begin{array}{l}
8562172331500 \\
97661475700 \\
473463600 \\
1218744 \\
1675 \\
1
\end{array}\right] .
$$

Equating the corresponding expressions and values of the column vectors $b_{1}$ and $b_{2}$, we obtain expressions for calculating $K_{P}, K_{I}, K_{D}$

$$
\begin{aligned}
& K_{I}=\frac{8562172331500}{5,3 \cdot 10^{10}}=160,54 ; \\
& K_{P}=\frac{97661475700-5,3 \cdot 10^{10}}{5,3 \cdot 10^{10}}=0,83 ; \\
& K_{D}=\frac{473463600-3,73 \cdot 10^{8}}{5,3 \cdot 10^{10}}=0,00188 .
\end{aligned}
$$

An obligatory condition for the implementation of the algorithm of the method under consideration is that the values of the PID controller coefficients must have the same sign, otherwise repeat step 3 of the procedure by specifying other values of the roots $s_{1}, s_{2}, s_{3}, s_{4}, s_{5}$ of the characteristic equation of the closed loop. In the example, all regulator parameters are positive - the test condition is met.

The system on Routh-Hurwitz criterion in obtained parameters is persistable. It is also approved by results of modeling in software MATLAB\&Simulink (Fig. 4).

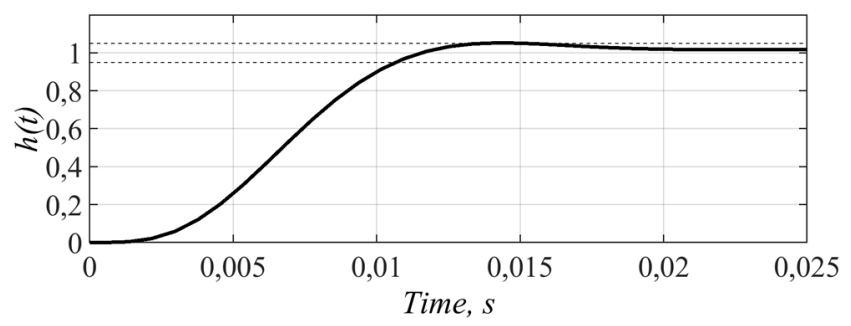

Fig. 4. Graph of the transient characteristic of the automatic control system with synthesized parameters of the PID control

Modeling of the transient characteristic of a control system with a PID controller parameters of which are analytically determined according to the algorithm of the proposed procedure with the example of a fourth-order object showed its compliance with the specified conditions by qualitative indicators - the regulation time and the nature of the transient process.

\section{CONCLUSIONS}

The method of parametric synthesis of a PID controller of a control system with feedback by high-order objects (above second) taking into account the placement of complex conjugate poles is considered. The method is implemented in the MathCAD software environment. Working capacity of the method showed good results corresponding to the design task on the example of the ACS model by the fourth order object. Transient process was without excessive correction.

\section{REFERENCES}

[1] D. Serpanos, M. Wolf, Internet-of-Things (IoT) Systems. Architectures, Algorithms, Methodologies. Springer, Cham, 2018, 95 p.

[2] A.P. Prokopiev, V.I. Ivanchura, R.T. Emelyanov, "Synthesis pid controller for objects second order with regard to the location poles", J. Sib. Fed. Univ. Eng. technol., 9(1), 2016, pp. 50-60.

[3] J.G. Ziegler, N.B. Nichols, "Optimum settings for automatic controllers”, Trans. Am. Soc. Mech. Eng. 64(11), 1942, pp. 759-768

[4] M. Johnson, "PID Control Technology", In: Johnson M.A., Moradi M.H. (eds) PID Control. Springer, London, 2005, pp. 1-46.

[5] K. Astrom, T. Hagglund, "The future of PID control. Control Engineering Practice", 9(11), 2001, pp. 1163-1175.

[6] C.L. Royce, H.D.Phillips, Feedback Control Systems. Pearson, 2010, 784 p.

[7] A. Visioli, PID Controllers for Time-Delay Systems, Springer-Verlag London, 2006, $314 \mathrm{p}$.

[8] P. Shah, S. Agashe, "Design of controller for a higher order system without using model reduction methods", Prog. Frac. Differ. Appl. Int. J., 3(4), 2017, pp. 289-304.

[9] A. O'Dwyer, Handbook of PI and PID Controller Tuning Rules, London, Imperial College Press, 2006, 564 p.

[10] A. O'Dwyer, Handbook of PI and PID controller tuning rules. London: Imperial College Press, 2009, 623 p.

[11] W. Hu, X. Yang, M. Zhu, Y. Zhang, "The Application of Automatic Control Theory in Software Engineering", In: Ma M. (eds) Communication Systems and Information Technology, Lecture Notes in Electrical Engineering, vol. 100, 2011, pp. 965-968.

[12] Li Shiping, , Hu Chunhua, "Study on Dynamic Model of Vibratory Roller - Soil System", IOP Conf. Series, Earth and Environmental Science, 2018, 113, 2018, 012187.

[13] Fang Xiaojun, Bian Yongming, Yang Meng, Liu Guangjun, "Development of a path following control model for an unmanned vibratory roller in vibration compaction", Advances in Mechanical Engineering, 10(5), 2018, pp. 1-16.

[14] B. Yongming, F. Xiaojun, Y. Meng, Z. Zhu, "Automatic Rolling Control for Unmanned Vibratory Roller Based on Fuzzy Algorithm", Journal of Tongji University (Natural Science), 45(12), 2017, pp. 1831-1838.

[15] D. White, M. Thompson, "Relationships Between In Situ and RollerIntegrated Compaction Measurements for Granular Soils", Journal of Geotechnical and Geoenvironmental Engineering, 134(12), 2008, pp. $1763-1770$

[16] S. Bejan, "The Roller-Ground Dynamic Interaction in The Compaction Process Through Vibrations for Road Construction", Romanian Journal of Transport Infrastructure, 5(2), 2016, pp. 1-9.

[17] F. Beainy, S. Commuri, M. Zaman, "Dynamical response of vibratory rollers during the compaction of asphalt pavements", J. Eng. Mech., 140(7), 2014.

[18] S.A. Imran, S. Commuri, M. Barman, M. Zaman, F. Beainy, "Modeling the dynamics of asphalt-roller interaction during compaction", Journal of Construction Engineering and Management, 143(7), 2017, pp. $1763-1770$ 
[19] Jian Sun, Guiyun Xu, "Dynamics Modeling and Analysis of Paver Screed Based on Computer Simulation", Journal of Applied Sciences, 13, 2013, pp. 1059-1065.

[20] K.J. Astrom, T. Hagglund, PID Controllers: Theory, Design, and Tuning, 2nd edn. ISA, 1995.
[21] María M. Seron, Julio H. Braslavsky, Goodwin Graham C.: Fundamental Limitations in Filtering and Control. Berlin: Springer, 1997, 369 p.

[22] Ogata Katsuhiko, Modern Control Engineering, Prentice Hall, 2010, $905 \mathrm{p}$. 